\title{
30-Y FOLLOW-UP OF A PU/AM INHALATION CASE
}

Christian Wernli ${ }^{1} *$, Jost Eikenberg ${ }^{1}$, Olaf Marzocchi ${ }^{2}$, Bastian Breustedt ${ }^{2}$, Ursula Oestreicher ${ }^{3}$, Horst Romm ${ }^{3}$, Demetrio Gregoratto ${ }^{4}$ and James Marsh ${ }^{4}$

${ }^{1}$ Paul Scherrer Institute (PSI), Villigen, Switzerland

${ }^{2}$ Karlsruhe Institute for Technology (KIT), Karlsruhe, Germany

${ }^{3}$ Federal Office for Radiation Protection (BfS), Oberschleissheim, Germany

${ }^{4}$ Public Health England (PHE), Harwell Science and Innovation Campus, UK

*Corresponding author: christian.wernli@alumni.ethz.ch

In 1983, a young man inhaled accidentally a large amount of plutonium and americium. This case was carefully followed until 2013. Since no decorporation measures had been taken, the undisturbed metabolism of $P u$ and Am can be derived from the data. First objective was to determine the amount of inhaled radionuclides and to estimate committed effective dose. In vivo and excretion measurements started immediately after the inhalation, and for quality assurance, all types of measurements were performed by different labs in Europe and the USA. After dose assessment by various international groups were completed, the measurements were continued to produce scientific data for model validation. The data have been analysed here to estimate lung absorption parameter values for the inhaled plutonium and americium oxide using the proposed new ICRP Human Respiratory Tract Model. As supplement to the biokinetic modelling, biological data from three different cytogenetic markers have been added. The estimated committed effective dose is in the order of $1 \mathrm{~Sv}$. The subject is $30 \mathrm{y}$ after the inhalation, of good health, according to a recent medical check-up.

\section{HISTORY OF THE INHALATION CASE}

A first paper describing the details of the accident, the early measurements and dose estimates of the $\mathrm{Pu} / \mathrm{Am}$ inhalation case of 1983 at the former Federal Institute for Reactor Research in Switzerland has been published in $2007^{(1)}$. Immediately after accidental inhalation by a 26 -y-old male technician, dose estimates were of primary interest. The results of measurements performed in various labs were used by several organisations for testing their internal dosimetry programmes for actinides. Later on, this case became mainly of scientific interest since no chelating agent was used and, even after $30 \mathrm{y}$, in vivo and excretion measurements were still possible. Fortunately, the person concerned accepted all these measurement procedures. Over the last few years, organ measurements were performed with highly specialised instruments at Karlsruhe Institute for Technology (KIT) ${ }^{(2)}$ and the excretion measurements were done again at Paul Scherrer Institute (PSI $^{(1)}$. In addition to all the physical measurements, current cytogenetic analyses were performed at Federal Office for Radiation Protection ${ }^{(3)}$ to complete scientific data by biological studies.

\section{RECENT IN VIVO MEASUREMENTS}

\section{Instruments and method}

Recent in vivo measurements have been performed at KIT Karlsruhe using a system of four HPGe detectors $^{(2)}$. Two detector configurations were used: a mixed one to monitor four organs (left lung, right lung, liver and knee), and a configuration specific for the skeleton (two detectors around the skull and two detectors for the knees). The measurement time was $4000 \mathrm{~s}$ per configuration. The net area of the peaks was estimated according to ISO 28218 . The calculation of the activity for ${ }^{241} \mathrm{Am}$ in each organ was performed using both ICRP Man and ICRP Female as calibration phantoms, and the calibration data were obtained using MCNPX $^{(4)}$ after a validation of the $\operatorname{method}^{(2)}$. The analysis of the data was performed first for the mixed configuration; the results were used to correct the calculations for the skeleton configuration.

The calculation of the activities in the mixed configuration was not performed using each detector independently, because it is known that a measurable part of the counts is generated by the crosstalk between neighbouring organs and detectors. Instead, the direct and the indirect contributions and the four peak areas were used to write a system of linear equations. The solution of the system consisted of the activity in each organ.

\section{DISCUSSION}

The solution of the system of equations applied to the mixed measurement configuration produced a negative value of ${ }^{241} \mathrm{Am}$ activity in the liver, independently from the calibration phantom used, suggesting the lack of ${ }^{241} \mathrm{Am}$ in the organ. The system was therefore rewritten to exclude the liver and the solution calculated again. The final data are shown in Table 1.

The activity in the lungs calculated using the mixed configuration was used to correct the peaks recorded in the detectors around the skull. This was performed 


\section{WERNLI ET AL}

by simulating with MCNPX the counting efficiency for photons originated in the lungs and reaching the detectors around the skull. The number of counts originated by the skeleton was therefore lowered: the net value was 3-5\% lower when using ICRP Male as calibration phantom and 8-10\% lower for ICRP Female. The different results can be explained by the higher amount of muscles in ICRP Male that acts as shield and lowers the cross-counting efficiency for the detectors around the skull. The corrected activities are listed in Table 2.

By comparing the results for the mixed detector configuration with the results for the skeleton configuration, it appears that the activity value for the skeleton is coherent only when using ICRP Female: the calibration with ICRP Man produces a discrepancy of $\sim 30 \%$.

A partial explanation for the issue is related to the MCNPX simulations and the quality of the models used to reproduce the detectors: currently, they are optimised only for the front face, not for the photons impinging on the sides of the crystal. More accurate models will be available in the future.

The placement of the detectors relative to the subject affects the results heavily, and it is the most probable cause of the discrepancy between skull and knees measurements. Additional simulations found the efficiency for the skull to decrease by $\sim 50 \%$ for a $2-\mathrm{cm}$ slide of the subject down the reclined stretcher [see the pictures in (2)]. The placement of the detectors was checked at the beginning, but not at the end of the measurement sessions; therefore, an accurate correction factor for the measurements around the

Table 1. Activities calculated for the mixed configuration, according to the calibration phantom used.

\begin{tabular}{lcc}
\hline Organ & ICRP Female [Bq] & ICRP Man [Bq] \\
\hline Skeleton $^{\mathrm{a}}$ & 152 & 288 \\
Lung left $^{\mathrm{b}}$ & 60 & 73 \\
Lung right $^{\mathrm{b}}$ & 59 & 77 \\
Liver $^{\mathrm{a}}$ & 0 & 0 \\
\hline
\end{tabular}

${ }^{a}$ Based on knee measurements.

${ }^{\mathrm{b}}$ The calibration phantom did not distinguish between lung and thoracic lymph nodes.

Table 2. Activities calculated for the skeleton-specific configuration, according to the calibration phantom used.

\begin{tabular}{lcc}
\hline Organ measured & ICRP Female [Bq] & ICRP Man [Bq] \\
\hline Knee & 103 & 216 \\
Knee & 141 & 270 \\
Average knees & 122 & 243 \\
Skull left & 176 & 556 \\
Skull right & 184 & 449 \\
Average skull & 180 & 503 \\
\hline
\end{tabular}

skull is not possible. A similar issue may affect the data for the knees, but the effect is an order of magnitude smaller: the simulations showed a decrease in the counting efficiency of at most $7 \%$ for a slide of $2 \mathrm{~cm}$.

\section{INTERPRETATION OF MEASUREMENTS}

This inhalation case has been analysed in the past by different authors and has been used in an internal dosimetry inter-comparison exercise ${ }^{(5)}$. The analysis did show a very long lung retention, which could only in part be accounted for by assuming that the inhaled material was very insoluble. A significantly slower particle transport clearance mechanism in the lungs had also to be assumed in order for the model predictions to agree with the measurements.

The data have been analysed here, by using the following models:

- for deposition, particle transport and absorption to blood in the respiratory tract, a revision of the Human Respiratory Tract Model (HRTM) ${ }^{(6)}$. In the revised particle transport model, the material deposited in the alveolar compartment clears to the bronchial tree at a rate of $m_{\mathrm{T}}=0.002 \mathrm{~d}^{-1}$ and to the interstitial compartment at a rate of $m_{\mathrm{I}}=0.001 \mathrm{~d}^{-1}$. The interstitial compartment clears very slowly to the regional lymph nodes at a rate of $0.00003 \mathrm{~d}^{-1}$;

- for transit through the alimentary tract the ICRP30 model $^{(7)}$;

- for systemic biokinetics, the Leggett model for plutonium $^{(8)}$ and the ICRP model for americium ${ }^{(9)}$.

The isotopic composition of the inhaled aerosol was estimated from measurements in January 1983 (4 months before the accident) on the fuel samples used in the solution that was overheated and spread out in the accident. The alpha activity composition is recorded as $10 \%{ }^{241} \mathrm{Am}, 9 \%{ }^{238} \mathrm{Pu}, 55 \%{ }^{239} \mathrm{Pu}$ and $26 \%{ }^{240} \mathrm{Pu}$. The ${ }^{241} \mathrm{Pu}$ (beta) activity is $750 \%$ of the total alpha activity. The uncertainty on these measurements is not known. The above isotopic composition is consistent with the activity ratio ${ }^{238} \mathrm{Pu}+$ $\left.{ }^{241} \mathrm{Am}\right) /\left({ }^{239} \mathrm{Pu}+{ }^{240} \mathrm{Pu}\right)=0.24 \pm 0.05$ calculated from the early faecal samples. There are no faecal measurements for ${ }^{241} \mathrm{Pu}$, but a previous laboratory record gives the ${ }^{241} \mathrm{Pu}$ activity as $655 \%$ of the total alpha activity whereas all the alpha activities are within a few per cent from the values given above. The reason of the discrepancy for ${ }^{241} \mathrm{Pu}$ is not known.

As in the previous analysis, an effective AMAD $^{(10)}$ (Activity Median Aerodynamic Diameter) of $\sim 5 \mu \mathrm{m}$ has been estimated from early lung and early faecal excretion data (including the activity that would have appeared in the faeces if it had not been not removed with a nasal swab and a bronchial slime before the first chest measurement was done $\left.{ }^{(1)}\right)$. This value has been used to calculate the fractions of material 
deposited in each of the lung regions. The calculated activity deposited in the nasal region has been adjusted by subtracting the amount that was removed immediately after the intake by nose swab. This correction does not affect the estimate of the effective dose but only the fit to the very early faecal data.

Particles deposited in the lungs are cleared by two competing mechanisms, by particle transport (to the gut and to the lymph nodes) and by absorption to blood of the dissolving material. For the latter, $f_{\mathrm{r}}$ indicates the fraction dissolved rapidly at the rate $s_{\mathrm{r}}$, whereas the complementary fraction $\left(1-f_{\mathrm{r}}\right)$ is dissolved slowly at the rate $s_{\mathrm{s}}$. A fraction $f_{\mathrm{b}}$ of the dissolved material may not be absorbed directly into blood and could bind temporarily $\left(s_{\mathrm{b}}>0\right)$ or permanently $\left(s_{\mathrm{b}}=\right.$ 0 ) to lung tissues. As mentioned, the fraction $\left(1-f_{\mathrm{b}}\right)$ is available to be absorbed to blood at the rates $s_{\mathrm{r}}$ and $s_{\mathrm{s}}$.

Absorption of material from the respiratory tract to blood was shown to be slow in previous analysis. The inhaled material was initially assumed to be 'type S' solubility ${ }^{(11)}$, with absorption parameter values: $f_{\mathrm{r}}=$ $0.001, s_{\mathrm{r}}=1 \mathrm{~d}^{-1}, s_{\mathrm{s}}=0.001 \mathrm{~d}^{-1}$, and a bound state was assumed with $f_{\mathrm{b}}=0.002$ and $s_{\mathrm{b}}=0 \mathrm{~d}^{-1(6,12)}$. Note that the bound state does not play a significant role in the long-term retention in the lungs because the material is relatively insoluble. The gut uptake fraction was kept fixed to its default value of $f_{1}=5$ $10^{-4}$ for type $\mathrm{S}$ material.

Measurement errors were assumed to be log-normally distributed. The geometric standard deviations, or scattering factors, for the different datasets were either estimated from the data, as described by Marsh et al. ${ }^{(13)}$, or based on the default values given in the IDEAS guidelines $^{(10)}$. The values for the scattering factors were $1.2,1.6$ and 2.4 for chest, urine and faecal measurements and 2 for liver and skeleton.

The agreement between measurements and model predictions was poor when using default parameter values, and the following changes to the models were introduced to improve the fit to the data:

- the particle transport in the alveolar-interstitial region was reduced;

- the absorption parameter values of the inhaled material were optimised, either assuming that they were the same for $\mathrm{Am}$ and $\mathrm{Pu}$ (i.e. shared values) or that they were different (i.e. independent);

- the isotopic activity composition was allowed to vary;

- the transfer rates from blood to urinary bladder were varied in order to improve the fit to the skeleton and liver measurements.

The changes were applied by finding optimal model parameter values using the maximum-likelihood method. To address first the issue of activity balance, only chest and excretion data were used. Liver and skeleton data were included only in the last stage of
Table 3. Lung absorption parameters, committed effective dose (CED) and organ doses (equivalent doses and per cent contribution to the effective dose) for each added changes in the optimisation.

\begin{tabular}{llll}
\hline & $\begin{array}{c}\mathrm{PT}+ \\
\mathrm{AbsP}\end{array}$ & $\begin{array}{c}\text { + isotopic } \\
\text { composition }\end{array}$ & $\begin{array}{c}\text { Blood- } \\
\text { UB }\end{array}$ \\
\hline$f_{\mathrm{r}}(\mathrm{Pu})$ & 0.0014 & 0.0017 & 0.004 \\
$f_{\mathrm{r}}(\mathrm{Am})$ & 0.05 & 0.04 & 0.08 \\
$s_{\mathrm{r}}\left(\mathrm{d}^{-1}\right)$ & 0.21 & 0.19 & 0.32 \\
$s_{\mathrm{s}}\left(10^{-5} \mathrm{~d}^{-1}\right)$ & 5 & 5 & 7 \\
$\mathrm{CED}(\mathrm{Sv})$ & 1.3 & 1.1 & 1.2 \\
Lung & $8.3(77)$ & $6.8(74)$ & $6.3(67)$ \\
{$[$ Sv $(\%)]$} & & & $3(10)$ \\
Liver & $2.3(7)$ & $2(7)$ & $14(12)$ \\
Bone surface & $10(8)$ & $9(8)$ & $0.7(7)$ \\
R.B.M. & $0.5(5)$ & $0.5(5)$ & $212^{\mathrm{a}}$ \\
$\chi_{\text {TOT }}^{2}$ & 261 & 232 & 12 \\
$(236$ data $)$ & & & \\
$\chi_{\text {LUNG }}^{2}$ & 38 & 16 & \\
$(17$ data $)$ & & & \\
\hline
\end{tabular}

a242 data, including skeleton (3) and liver (3) data.

the optimisation. The agreement between model prediction and measurements improved significantly, in terms of chi-squared, for each of the above changes (Table 3).

The adoption of the revised HRTM model ${ }^{(6)}$ improves the fit to lung and faecal excretion data, compared with the previous $\mathrm{HRTM}^{(11)}$, but a further reduction of the particle transport rate from the alveolar region to the bronchiolar needs to be applied, from $m_{\mathrm{T}}=2 \times 10^{-3} \mathrm{~d}^{-1}$ to $m_{\mathrm{T}}=3 \times 10^{-4} \mathrm{~d}^{-1}$. The optimal value for the rate to the interstitial regions $\left(m_{\mathrm{I}}=8.5 \times 10^{-4} \mathrm{~d}^{-1}\right)$ differs only slightly from the default value $\left(m_{\mathrm{I}}=1 \times 10^{-3} \mathrm{~d}^{-1}\right)$. The changes may also be expressed as an increase of the so-called sequestered fraction in the alveolar-interstitial region, $m_{\mathrm{I}} /\left(m_{\mathrm{I}}+m_{\mathrm{T}}\right)$ from 0.33 to 0.74 and in the slowdown of the clearance to the gut. This value is relatively high but close to the upper limit of the $68 \%$ probability range for the inter-subject variation: $(0.2$, $0.7)^{(14)}$. Assuming that americium is more soluble than plutonium in the lungs improves the overall prediction for urine excretion, although the very late americium excretion remains slightly overestimated. The rapid and slow absorption rates $s_{\mathrm{r}}$ and $s_{\mathrm{s}}$ for plutonium are not well determined by the data, and it would be possible to assume the same values of $s_{\mathrm{r}}$ and $s_{\mathrm{S}}$ (shared values) for both $\mathrm{Am}$ and $\mathrm{Pu}$ without affecting significantly the fit to the data. However, a much better fit is obtained when the values for the rapid fraction $f_{\mathrm{r}}$ are optimised independently. The optimal shared values are $s_{\mathrm{r}}=0.2 \mathrm{~d}^{-1}$ and $s_{\mathrm{s}}=5 \times 10^{-5} \mathrm{~d}^{-1}$, and $f_{\mathrm{r}}=0.0014$ for $\mathrm{Pu}$ and $f_{\mathrm{r}}=0.05$ for Am. The result might be interpreted as an indication that 


\section{WERNLI ET AL}

initially americium dissolves faster. Americium oxide is generally more soluble than plutonium oxide ${ }^{(15)}$, but it is usually also considered that the rate at which a particle dissociates is determined by the particle matrix and therefore the dissolution parameter values for americium and plutonium should be similar if they belong to the same matrix. An interpretation of the above result could possibly be provided by a better knowledge of the physicochemical properties of the inhaled material.

The overall fit is not sufficiently good, and this is mainly due to the model prediction being 'pulled' in opposite directions by chest and urine data.

An estimate of the isotopic composition of the inhaled material is necessary to determine the initial amount of americium and plutonium (isotopes) deposited in the lungs. The values given above have been considered as fixed in previous analysis. Based on the available information, the authors assumed here a relative error of $10 \%$ and used it for a constrained optimisation. This would be equivalent to introduce a prior distribution for the isotopic fractions within a Bayesian framework.

The result of the optimisation, $13 \%{ }^{241} \mathrm{Am}, 9 \%$ ${ }^{238} \mathrm{Pu}, 53 \%{ }^{239} \mathrm{Pu}, 25 \%{ }^{240} \mathrm{Pu}$ and $660 \%{ }^{241} \mathrm{Pu}$, shows that the complete set of data and the models used might be more consistent with a different isotopic composition. However, the ratio $\left({ }^{238} \mathrm{Pu}+{ }^{241} \mathrm{Am}\right) /$ $\left({ }^{239} \mathrm{Pu}+{ }^{240} \mathrm{Pu}\right)=0.25$ is still compatible with the estimate $0.24 \pm 0.05$ based on the early faecal measurements. The lower beta activity $\left({ }^{241} \mathrm{Pu}\right)$ also cannot be completely excluded because of the two different values given in the laboratory records.

The previous adjustments to the models improve significantly the overall agreement between model prediction and experimental data, except for liver and skeleton. The reasons for the discrepancy could be various, but to improve the fit to the liver and skeleton data, it is necessary to modify systemic parameter values. A sensitivity analysis shows that reducing the amount transferred from blood to bladder is the most effective in ensuring a good fit to the two systemic organ datasets and improving further the agreement with the urine data. The main effect is an increase of the dose to internal organs, because more activity is retained in the body instead of being excreted through urine, and an adjustment of the lung absorption parameters. Note that this effect is achieved even if liver and skeleton data are not included in the fitting because it is mainly driven by the urine data. The rates from blood to urinary bladder have been changed by keeping constant the removal half-time from blood. A constraint equivalent to a lognormal prior distribution with geometric standard deviation equal to $\log (1.7)^{(16)}$ has been applied in the optimisation.

Table 3 shows how the optimal parameter values, doses and chi-squared did vary when the changes [particle transport (PT) plus absorption parameters (AbsP), isotopic composition, blood to urinary bladder] were applied sequentially.

\section{LUNG ABSORPTION PARAMETERS}

The maximum-likelihood procedure used for the parameters estimation indicates that the shared parameter $s_{\mathrm{r}}$ and $f_{\mathrm{r}}$ for $\mathrm{Pu}$ are less well defined by the data than the other parameters. The estimates of $f_{\mathrm{r}}, s_{\mathrm{r}}$ and $s_{\mathrm{s}}$ are not significantly correlated between them $(|\rho|<0.15)$ but show higher correlation with the estimate of the blood-to-urinary bladder rate.

The uncertainty on the estimates of the lung absorption parameters has been further investigated by using Bayesian Markov chain Monte Carlo sampling ${ }^{(17)}$. Lognormal prior distributions have been assumed for the parameters, which were constraint in the optimisation. 'Non-informative' priors (normal distribution for the log-transformed variable with $\sigma=2$ and centred on the best-fit result) have been used for all the other parameters. Most of the absorption parameters were well defined (Table 4 ) as the posterior distribution was relatively narrow (compared with the prior distribution) and approximately centred on the best-fit value. The exceptions are the fast-absorbed fraction $f_{\mathrm{r}}$ for plutonium, for which only an upper limit $\left(f_{\mathrm{r}}<0.015\right)$ is clearly defined, and for the shared parameter $s_{\mathrm{r}}$ for which the lower limit is better defined than the upper limit $\left(s_{\mathrm{r}}>0.2\right.$ $\mathrm{d}^{-1}$ ). All set of measurements used in the analysis are given in the Annex (Tables A1-A5). Figures 1-4 show measurements and best-fit model predictions for a subset of the datasets. As shown in Figure 1, most of the ${ }^{241} \mathrm{Am}$ activity predicted in chest at later times is due to in-growth from ${ }^{241} \mathrm{Pu}$.

\section{CYTOGENETIC ANALYSIS}

In addition to the biokinetic modelling, three different cytogenetic assays have been performed to analyse diverse biomarkers of exposure in blood lymphocytes.

\section{Dicentric assay}

Up to now, the conventional analysis of dicentric chromosomes (dicentric assay) is the most appropriate

Table 4. MCMC results for lung absorption parameters and CED.

\begin{tabular}{lll}
\hline & Mean value & $95 \%$ probability interval \\
\hline$f_{\mathrm{r}}(\mathrm{Am})$ & 0.08 & $0.04-0.12$ \\
$f_{\mathrm{r}}(\mathrm{Pu})$ & 0.003 & $<0.015$ \\
$S_{\mathrm{r}}\left(\mathrm{d}^{-1}\right)$ & 0.4 & $0.17-5$ \\
$s_{\mathrm{s}}\left(\mathrm{d}^{-1}\right)$ & $8 \times 10^{-5}$ & $6 \times 10^{-5}-1 \times 10^{-4}$ \\
$\mathrm{CED}(\mathrm{Sv})$ & 1.2 & $1.0-1.35$ \\
\hline
\end{tabular}




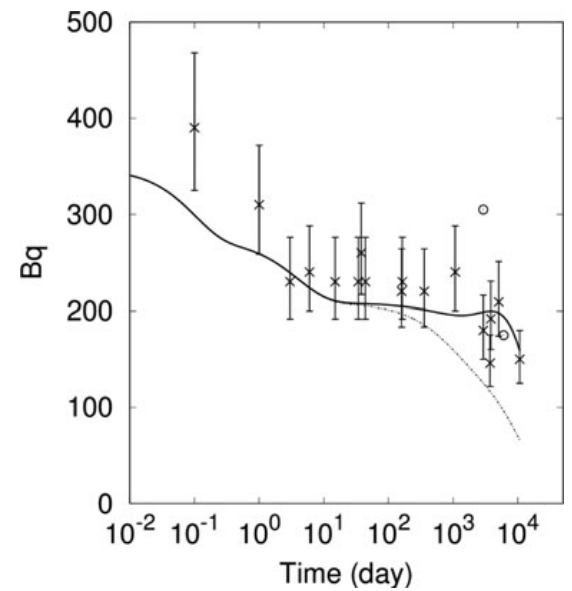

Figure 1. ${ }^{241} \mathrm{Am}$ in chest. The dash-dotted curve shows the predicted ${ }^{241} \mathrm{Am}$ activity without taking into account of in-growth from ${ }^{241} \mathrm{Pu}$.

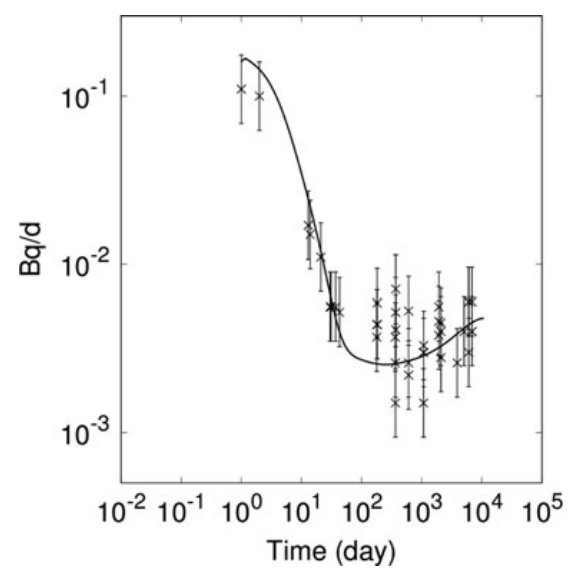

Figure 2. ${ }^{241} \mathrm{Am}+{ }^{238} \mathrm{Pu}$ in 24 -h urine excretion.

assay to estimate a dose in case of an acute irradiation ${ }^{(18,19)}$. This assay was also used in a previous examination of the same person years ago ${ }^{(1)}$. In the present study, a total of 1000 cells from Giemsa stained slides were analysed. The observed frequency of 2 dicentric chromosomes per 1000 cells was not significantly different $(p>0.05)$ in comparison with the authors' control value of 1.15 dicentric chromosomes per 1000 cells. This result was to be expected because of the 30-y time period between the first accidental exposure and the current blood sampling. The biological half-life of lymphocytes with dicentric chromosomes is assumed to be $3 \mathrm{y}$. Due to a detriment of dicentric chromosomes during cell division, the yield of lymphocytes in the circulating blood bearing this biomarker will decrease in the course of time.

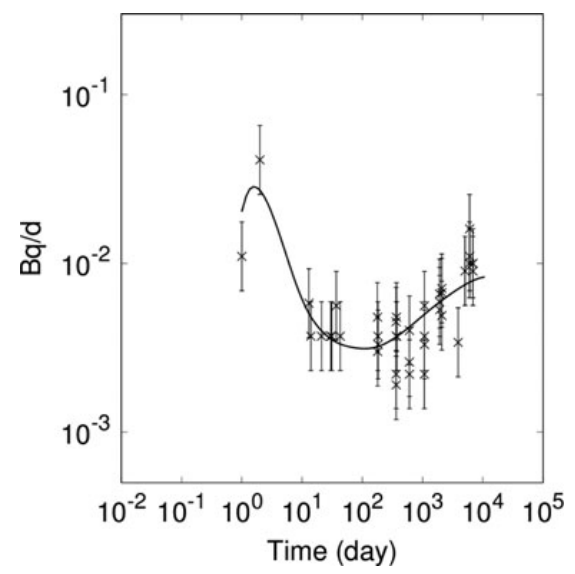

Figure 3. ${ }^{239} \mathrm{Pu}+{ }^{240} \mathrm{Pu}$ in $24-\mathrm{h}$ urine excretion.

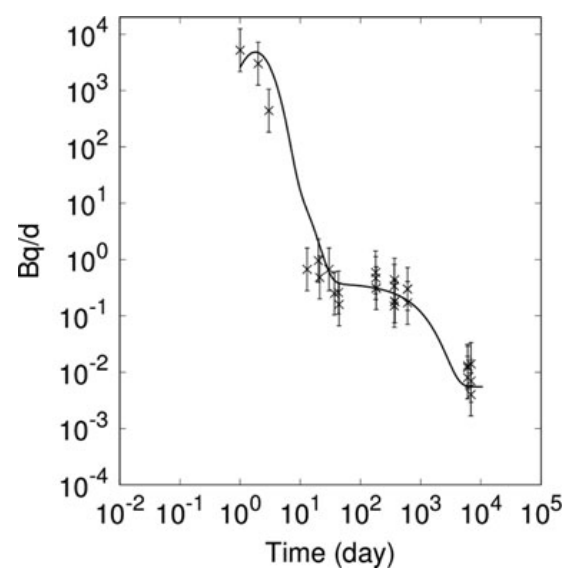

Figure 4. ${ }^{239} \mathrm{Pu}+{ }^{240} \mathrm{Pu}$ in $24-\mathrm{h}$ faecal excretion.

\section{FISH assay (symmetrical translocations)}

The yield of symmetrical translocations in 3017 cells scored was significantly increased $(23.8 \pm 5.0 / 1000$ cells, FG (Genome equivalents) values) in comparison with an age-adjusted control group including 35 persons and 88934 cells scored [7.5 $\pm 0.51 / 1000$ cells, FG (Genome equivalent) values]. This aberration type has the advantage to pass cell division without major detriment and thus is more persistent. In consequence, symmetrical aberrations are the indicator of choice for past and chronic radiation conditions ${ }^{(20)}$. In the current case, the significant increase of symmetrical translocations indicates an irradiation incident. Because of the lack of an appropriate dose-effect curve for this radiation quality (alpha particles) and the extreme long time period since the exposure, a dose reconstruction was considered to be not feasible. 


\section{WERNLI ET AL}

\section{Micronucleus assay}

For micronuclei, the increase observed in comparison with the unexposed control group was ambiguous. In fact, there was a significant increase in the mean value of micronuclei compared with the control value; however, there still was an overlap with the upper dose interval of the non-irradiated control. The reason is the relatively high and variable spontaneous frequency of micronuclei in control cells, which tends to increase with age and also shows gender dependency $^{(21)}$. Additionally problematic is the lack of specificity of micronuclei, as they can also be caused by chemical clastogenic agents. Therefore, the increase found here cannot clearly be assigned to the radiation accident; however, a relationship cannot be excluded.

In summary, the significant increase of symmetrical aberrations, while no enhancement of dicentric chromosomes is observed, provides evidence for a past radiation exposure of the blood forming tissue and/or ongoing chronic low dose exposure.

\section{CONCLUSION AND OUTLOOK}

The $\mathrm{Pu} / \mathrm{Am}$ inhalation case of 1983 has been studied by colleagues in several institutions and described in numerous papers including the publication of $2007^{(1)}$, the actual publication and many papers cited in both publications. The aim of the full publication of all data of this case is to make this information available for internal dosimetry model evaluation and training in internal dosimetry. It will depend on the availability and willingness of the person involved whether the series of measurements can be continued and the results presented in a future update.

\section{ACKNOWLEDGEMENTS}

The authors thank the person who has suffered the $\mathrm{Pu} / \mathrm{Am}$ inhalation in 1983 for his continued willingness to cooperate in all the studies performed. They also thank the medical service of the Swiss Social Insurance Fund (Suva) for the recent general medical check-up, which fortunately attested good health of the person.

\section{REFERENCES}

1. Wernli, C. and Eikenberg, J. Twenty-year-follow-up of a $\mathrm{Pu} /$ Am inhalation case. Radiat. Prot. Dosim. 125(1-4), 506-512 (2007).

2. Marzocchi, O. Design and setup of a new HPGe detector based body counter capable of detecting also low energy photon emitters. University of Bologna, Thesis. Chapters $4.3 ; 5 ; 6.2,(2011)$.

3. Stephan, G., Oestreicher, U. and Romm, H. Biological dosimetry. In: Chromosomal Alterations-Methods, Results and Importance in Human Health. Obe, G. and Vijayalaxmi, Eds. Springer, pp. 341 - 349 (2007).
4. Pelowitz, D. B. (ed.), MCNPX User's Manual Version 2.7.0. Los Alamos National Laboratory, (2011). LACP-11-00438.

5. IAEA. IAEA-TECDOC-1568. Intercomparison Exercise on Internal Dose Assessment. IAEA, (2007).

6. ICRP. Occupational intakes of radionuclides, Part 1. Annals of the International Commission on Radiological Protection, Accepted for publication, (2014a).

7. ICRP. Limits for intakes of radionuclides by workers ICRP publication 30 (Part 1). Ann. ICRP 2 (3-4), (1979).

8. Leggett, R. W. et al. Mayak worker study: an improved biokinetic model for reconstructing doses from internally deposited plutonium. Radiat. Res. 164(2), 111-122 (2005).

9. ICRP. Age-dependent doses to members of the public from intake of radionuclides: Part 2. Ingestion dose coefficients. A report of a Task Group of Committee 2 of the International Commission on Radiological Protection. Annals of the ICRP, 23(3-4), pp. 1-167 (1993).

10. Castellani, C., Marsh, J. and Hurtgen, C. IDEAS Guidelines (Version 2) for the estimation of committed doses from incorporation monitoring data. (2013). Available on http://www.eurados.org/ /media/Files/Eura dos/documents/EURADOS $\% 20$ Report $\% 202013-01 \% 20$ online $\% 20$ version.pdf [Accessed June 27, 2014].

11. ICRP. Human respiratory tract model for radiological protection. A report of a Task Group of the International Commission on Radiological Protection. Annals of the ICRP, 24(1-3), pp. 1-482 (1994).

12. ICRP. Occupational intakes of radionuclides, Part 4. Annals of the International Commission on Radiological Protection, in progress (2014).

13. Marsh, J. W. et al. Evaluation of scattering factor values for internal dose assessment following the IDEAS guidelines: preliminary results. Radiat. Prot. Dosim. 127(1-4), 339-342 (2007).

14. Gregoratto, D., Bailey, M. R. and Marsh, J. W. Modelling particle retention in the alveolar-interstitial region of the human lungs. J. Radiol. Prot. 30(3), 491-512 (2010).

15. ICRP. Dose coefficients for intakes of radionuclides by workers. ICRP Publication 68. Ann. ICRP 24 (4) (1994b).

16. Puncher, M. and Harrison, J. D. Uncertainty analysis of doses from ingestion of plutonium and americium. Radiat. Prot. Dosim. 148, 284-296 (2012).

17. Gelman, A. and Rubin, D. B. Markov chain Monte Carlo methods in biostatistics. Stat. Methods Med. Res. 5(4) 339-355 (1996).

18. International Atomic Energy Agency. Cytogenetic dosimetry: applications in preparedness for and response to radiation emergencies. EPR-Biodosimetry 2011. IAEA (2011).

19. Romm, H., Oestreicher, U. and Kulka, U. Cytogenetic damage analysed by the dicentric assay. In: Biodosimetric tools for a fast triage of people accidentally exposed to ionising radiation. Ann. Ist Super Sanità. 45, 251-259 (2009).

20. Ainsbury, E. A., Bakhanova, E., Barquinero, J. F., Brai, M., Chumak, V., Correcher, V., Darroudi, F., Fattibene, P., Gruel, G., Guclu, I. et al. Retrospective dosimetry techniques for external radiation exposures. Radiat. Prot. Dosim. 147, 573-592 (2011).

21. Fenech, M. The cytokinesis-block micronucleus technique: a detailed description of the method and its application to genotoxicity studies in human populations. Mutat. Res. 285, 35-44 (1993). 
ANNEX

Table A1. Chest ${ }^{\mathrm{a}}$, faeces and urine measurements: ${ }^{241} \mathrm{Am}$.

\begin{tabular}{|c|c|c|c|c|c|}
\hline \multicolumn{2}{|l|}{ Chest } & \multicolumn{2}{|c|}{ Faeces } & \multicolumn{2}{|c|}{ Urine } \\
\hline$t(\mathrm{~d})$ & $\mathrm{Bq}$ & $t(\mathrm{~d})$ & $\mathrm{Bq} \mathrm{d}^{-1}$ & $t(\mathrm{~d})$ & $\mathrm{Bq} \mathrm{d}^{-1}$ \\
\hline 0.1 & 390 & 1074 & 0.0180 & 2092 & 0.0023 \\
\hline 1 & 310 & 1075 & 0.0300 & 2095 & 0.0036 \\
\hline 3 & 230 & 1076 & 0.0170 & 2525 & $<0.0015$ \\
\hline 6 & 240 & 1077 & 0.0070 & 2526 & 0.0051 \\
\hline 15 & 230 & 1921 & 0.0130 & 2527 & 0.0034 \\
\hline 34 & 230 & 1922 & 0.0460 & 2921 & 0.0027 \\
\hline 38 & 260 & 1923 & 0.0150 & 2922 & 0.0019 \\
\hline 44 & 230 & 2525 & 0.0170 & 2923 & $<0.0015$ \\
\hline 160 & 220 & 2526 & 0.0050 & 6043 & 0.0050 \\
\hline 164 & 230 & 2527 & 0.0130 & 6044 & 0.0020 \\
\hline 357 & 220 & 2921 & $<0.0015$ & 6045 & 0.0050 \\
\hline 1077 & 240 & 2922 & 0.0060 & 6859 & 0.0050 \\
\hline 2925 & 180 & 2923 & $<0.0015$ & 6860 & 0.0030 \\
\hline 2926 & $305^{b, c}$ & 6043 & 0.0040 & 6861 & 0.0030 \\
\hline 3724 & $146^{\mathrm{c}}$ & 6044 & 0.0040 & 6870 & 0.0030 \\
\hline 3828 & $192^{c}$ & 6045 & 0.0020 & 6903 & 0.0021 \\
\hline 5078 & $209^{c}$ & 6859 & 0.0010 & 10708 & 0.0049 \\
\hline 6037 & $175^{b, c}$ & 6860 & 0.0020 & 10709 & 0.0019 \\
\hline \multirow[t]{4}{*}{10713} & $150^{\mathrm{d}}$ & 6861 & 0.0020 & 10710 & 0.0016 \\
\hline & & 10708 & 0.0023 & & \\
\hline & & 10709 & 0.0004 & & \\
\hline & & 10710 & 0.0021 & & \\
\hline
\end{tabular}

${ }^{a}$ Chest measurements may include counts from thoracic lymph nodes, ribs and liver.

${ }^{\mathrm{b}}$ Data that have been excluded from the fitting.

${ }^{\mathrm{c}}$ Lung and lymph node activities were measured separately and added together.

${ }^{\mathrm{d}}$ See text for description of measurement.

Table A2. Liver and skeleton measurements: ${ }^{241} \mathrm{Am}$.

\begin{tabular}{|c|c|c|c|}
\hline \multicolumn{2}{|l|}{ Liver } & \multicolumn{2}{|c|}{ Skeleton } \\
\hline$t(\mathrm{~d})$ & $\mathrm{Bq}$ & $t(\mathrm{~d})$ & $\mathrm{Bq}$ \\
\hline 2926 & 27 & 2926 & 57 \\
\hline 3724 & $57^{\mathrm{a}}$ & 3724 & 69 \\
\hline 3828 & 24 & 3828 & 65 \\
\hline \multirow[t]{2}{*}{5078} & 17 & 5078 & 95 \\
\hline & & 6037 & 95 \\
\hline 10713 & 0 & 10713 & $243^{\mathrm{a}}$ \\
\hline
\end{tabular}

${ }^{\mathrm{a}}$ Data that have been excluded from the fitting.
Table A3. Faeces and urine measurements: ${ }^{238} \mathrm{Pu}+{ }^{241} \mathrm{Am}$.

\begin{tabular}{|c|c|c|c|}
\hline \multicolumn{2}{|c|}{ Faeces } & \multicolumn{2}{|c|}{ Urine } \\
\hline$t(\mathrm{~d})$ & $\mathrm{Bq} \mathrm{d}^{-1}$ & $t(\mathrm{~d})$ & $\mathrm{Bq} \mathrm{d}^{-1}$ \\
\hline 1 & 1500.000 & 1 & 0.1100 \\
\hline 2 & 740.000 & 2 & 0.1000 \\
\hline 3 & 74.000 & 13 & 0.0170 \\
\hline 13 & 0.160 & 14 & 0.0150 \\
\hline 20 & 0.190 & 21 & 0.0110 \\
\hline 21 & 0.110 & 30 & 0.0056 \\
\hline 30 & 0.120 & 31 & 0.0056 \\
\hline 37 & 0.078 & 37 & 0.0056 \\
\hline 43 & 0.074 & 43 & 0.0052 \\
\hline 44 & 0.044 & 179 & 0.0037 \\
\hline 179 & 0.120 & 180 & 0.0044 \\
\hline 180 & 0.100 & 182 & 0.0059 \\
\hline 182 & 0.093 & 183 & 0.0044 \\
\hline 183 & 0.063 & 364 & 0.0037 \\
\hline 365 & 0.081 & 365 & 0.0026 \\
\hline 366 & 0.026 & 366 & 0.0015 \\
\hline 369 & 0.110 & 369 & 0.0052 \\
\hline 370 & 0.037 & 370 & 0.0071 \\
\hline 371 & 0.041 & 371 & 0.0041 \\
\hline 605 & 0.085 & 605 & 0.0026 \\
\hline 606 & 0.080 & 606 & 0.0053 \\
\hline 607 & 0.059 & 607 & 0.0022 \\
\hline 6043 & 0.005 & 1074 & 0.0015 \\
\hline 6044 & 0.005 & 1075 & 0.0033 \\
\hline 6045 & 0.002 & 1076 & 0.0030 \\
\hline 6859 & 0.001 & 1077 & 0.0030 \\
\hline 6860 & 0.003 & 1921 & 0.0056 \\
\hline 6861 & 0.003 & 1922 & 0.0046 \\
\hline & & 1923 & 0.0038 \\
\hline & & 2089 & 0.0045 \\
\hline & & 2090 & 0.0040 \\
\hline & & 2091 & 0.0028 \\
\hline & & 3902 & 0.0026 \\
\hline & & 5076 & 0.0040 \\
\hline & & 6043 & 0.0060 \\
\hline & & 6044 & 0.0030 \\
\hline & & 6045 & 0.0060 \\
\hline & & 6859 & 0.0060 \\
\hline & & 6860 & 0.0040 \\
\hline & & 6870 & 0.0040 \\
\hline
\end{tabular}


C. WERNLI ET AL.

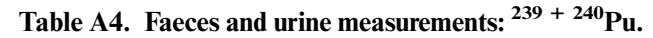

\begin{tabular}{|c|c|c|c|}
\hline \multicolumn{2}{|c|}{ Faeces } & \multicolumn{2}{|c|}{ Urine } \\
\hline$t(\mathrm{~d})$ & $\mathrm{Bq} \mathrm{d}^{-1}$ & $t(\mathrm{~d})$ & $\mathrm{Bq} \mathrm{d}^{-1}$ \\
\hline 1 & 5200.000 & 1 & 0.0110 \\
\hline 2 & 3000.000 & 2 & 0.0410 \\
\hline 3 & 440.000 & 13 & 0.0058 \\
\hline 13 & 0.670 & 14 & 0.0037 \\
\hline 20 & 0.960 & 21 & 0.0037 \\
\hline 21 & 0.480 & 30 & 0.0037 \\
\hline 30 & 0.670 & 31 & 0.0037 \\
\hline 37 & 0.250 & 37 & 0.0056 \\
\hline 43 & 0.260 & 43 & 0.0037 \\
\hline 44 & 0.160 & 179 & 0.0048 \\
\hline 179 & 0.590 & 180 & 0.0030 \\
\hline 180 & 0.470 & 182 & 0.0037 \\
\hline 182 & 0.310 & 183 & 0.0033 \\
\hline 183 & 0.310 & 364 & 0.0045 \\
\hline 365 & 0.340 & 365 & 0.0022 \\
\hline 366 & 0.150 & 366 & 0.0019 \\
\hline 369 & 0.440 & 369 & 0.0037 \\
\hline 370 & 0.180 & 370 & 0.0048 \\
\hline 371 & 0.180 & 371 & 0.0037 \\
\hline 605 & 0.300 & 605 & 0.0026 \\
\hline 606 & 0.300 & 606 & 0.0040 \\
\hline 607 & 0.170 & 607 & 0.0022 \\
\hline 6043 & 0.012 & 1074 & 0.0022 \\
\hline 6044 & 0.013 & 1075 & 0.0037 \\
\hline 6045 & 0.008 & 1076 & 0.0033 \\
\hline 6859 & 0.004 & 1077 & 0.0056 \\
\hline 6860 & 0.014 & 1921 & 0.0059 \\
\hline \multirow[t]{13}{*}{6861} & 0.007 & 1922 & 0.0053 \\
\hline & & 1923 & 0.0066 \\
\hline & & 2089 & 0.0071 \\
\hline & & 2090 & 0.0049 \\
\hline & & 2091 & 0.0067 \\
\hline & & 3902 & 0.0034 \\
\hline & & 5076 & 0.0090 \\
\hline & & 6043 & 0.0110 \\
\hline & & 6044 & 0.0100 \\
\hline & & 6045 & 0.0160 \\
\hline & & 6859 & 0.0100 \\
\hline & & 6860 & 0.0100 \\
\hline & & 6870 & 0.0090 \\
\hline
\end{tabular}

Table A5. Faeces and urine measurements: ${ }^{238+239+240} \mathrm{Pu}$.

\begin{tabular}{|c|c|c|c|}
\hline \multicolumn{2}{|c|}{ Faeces } & \multicolumn{2}{|c|}{ Urine } \\
\hline$t(\mathrm{~d})$ & $\mathrm{Bq} \mathrm{d}^{-1}$ & $t(\mathrm{~d})$ & $\mathrm{Bq} \mathrm{d}^{-1}$ \\
\hline 1074 & 0.0700 & 2525 & 0.0042 \\
\hline 1075 & 0.1200 & 2526 & 0.0064 \\
\hline 1076 & 0.0650 & 2527 & 0.0070 \\
\hline 1077 & 0.0250 & 2921 & 0.0047 \\
\hline 1921 & 0.0900 & 2922 & 0.0044 \\
\hline 1922 & 0.1900 & 2923 & $<0.0015^{\mathrm{a}}$ \\
\hline 1923 & 0.1000 & 3723 & 0.0100 \\
\hline 2525 & 0.0470 & 3725 & 0.0090 \\
\hline 2526 & 0.0170 & 6043 & 0.0130 \\
\hline 2527 & 0.0380 & 6044 & 0.0100 \\
\hline 2921 & 0.0050 & 6045 & 0.0170 \\
\hline 2922 & 0.0130 & 6859 & 0.0100 \\
\hline 2923 & 0.0030 & 6860 & 0.0100 \\
\hline 3723 & 0.0150 & 6861 & 0.0090 \\
\hline 3725 & 0.0110 & 6870 & 0.0100 \\
\hline 6043 & 0.0130 & 6903 & 0.0090 \\
\hline 6044 & 0.0140 & 10708 & 0.0090 \\
\hline 6045 & 0.0090 & 10709 & 0.0054 \\
\hline 6859 & 0.0040 & 10710 & 0.0040 \\
\hline 6860 & 0.0140 & & \\
\hline 6861 & 0.0080 & & \\
\hline 10708 & 0.0060 & & \\
\hline 10709 & 0.0017 & & \\
\hline 10710 & 0.0063 & & \\
\hline
\end{tabular}

${ }^{\mathrm{a}} \mathrm{Data}$ that have been excluded from the fitting. 